\title{
MicroRNA- 15la-3p Functions in the Regulation of Osteoclast Differentiation: Significance to Postmenopausal Osteoporosis
}

\section{Yuehui $\mathrm{He}$ \\ Di Chen \\ Qian Guo \\ Pinghua Shi \\ Conglei You \\ Yanping Feng}

Community Medicine Department, Beijing Jishuitan Hospital, Beijing City, 100096, People's Republic of China
Correspondence: Di Chen Community Medicine Department, Beijing Jishuitan Hospital, No. 68, Huinanbei Road, Huilongguan Town, Changping District, Beijing City, 100096, People's Republic of China

Email zr4560@I63.com
Background: Studies have found the pivotal role of miRNAs in the progression of postmenopausal osteoporosis (OP). However, the function of miRNAs in OP is unclear. This study aimed to explore the biological functions of microRNA-151a-3p in OP.

Methods: RT-qPCR was employed to assess the expression of microRNA-151a-3p in serum isolated from OP patients and healthy controls. Dual-energy X-ray absorptiometry (DXA) was used to measure the bone mineral density (BMD) of the lumbar spine. The expression levels of c-Fos, NFATc1, and TRAP were tested by Western blot. Ovariectomized (OVX) rats were treated with antago microRNA-151a-3p or antago $\mathrm{NC}$, and then serum and lumbar vertebrae were collected for ELISA and bone histomorphology analysis.

Results: The expression of microRNA-151a-3p in postmenopausal women with osteoporosis was significantly up-regulated, and microRNA-151a-3p level was negatively correlated with BMD. During osteoclastogenesis, microRNA-151a-3p level was obviously increased. Overexpression of microRNA-151a-3p promoted the differentiation of RANKL-induced THP-1 and RAW264.7 cells into osteoclasts, whereas silencing of microRNA-151a-3p resulted in the opposite results. Silencing of microRNA-151a-3p in OVX rats altered osteoclastogenesis-related factors and raised BMD.

Conclusion: MicroRNA-151a-3p could partly regulate osteoporosis by promoting osteoclast differentiation, and miRNA-151a-3p could be a potential therapeutic target for postmenopausal osteoporosis.

Keywords: miRNA-151a-3p, postmenopausal osteoporosis, osteoclast differentiation, RAW264.7 cells, THP-1 cells

\section{Background}

Postmenopausal osteoporosis (OP) is the most common type of osteoporosis. ${ }^{1}$ It is a systemic chronic bone disease without obvious clinical symptoms, and it is mainly caused by postmenopausal ovarian dysfunction, decrease of estrogen level, and degeneration of bone microstructure. ${ }^{2}$ The incidence of pathological fracture, a serious complication of OP, is more than $70 \%{ }^{3}$ With the rapid rise in the proportion of the elderly population, the incidence rate of OP has also increased significantly. It seriously affects the physical health and the quality of life of middle-aged patients. ${ }^{4}$ Physical activity not only contributes to a healthy energy balance and increased muscle and bone mass but also downregulates RANKL in bone cells. ${ }^{5,6}$ A variety of drugs have been used in the clinical treatment of OP, including estrogen, tamoxifen and bisphosphonate. However, due to the side 
effects, long course of treatment, inconvenient route of administration, and poor compliance of patients, the ideal curative effect cannot be achieved. ${ }^{7,8}$ Therefore, the prevention and treatment of OP has become a key issue to be solved urgently.

The primary cause of OP is the imbalance of the physiological process of bone turnover. ${ }^{9}$ The maturation of osteoclasts is regulated by multiple cytokines and signaling pathways, of which the NF- $\kappa \mathrm{B}$ pathway is the most important regulator. ${ }^{10,11}$ NFAT is another pathway involved in RANK in newly discovered osteoclasts. ${ }^{12}$ During osteoclast activation, titanium particles strongly trigger three major transcription factors, NFATc1, c-Fos and TRAP, which play key roles in the differentiation of osteoclast. ${ }^{13,14}$ Osteoclasts (OC) inhibit the formation and activation of OC by competing with RANK and binding to RANKL. Both TNF- $\alpha$ and IL- $1 \alpha$ can affect the expression of RANKL in osteoblasts, and regulate the formation and activation of OC. Recent studies have shown that TNF- $\alpha$ can induce bone marrow cells to differentiate into $\mathrm{OC}$ in the presence of M-CSF and CTX-I. However, the molecular mechanism of osteoclast differentiation in OP patients is not completely clear, therefore, understanding the regulatory mechanism of osteoclast differentiation in OP patients may be the key to improve the therapeutic effect of OP.

Almost all human chromosomes except for the Y chromosome express miRNAs. ${ }^{15}$ MiRNAs play key regulatory roles in cell division, apoptosis, signal transduction, and are involved in the progression of various diseases, such as cancer, cardiovascular disease, osteoarthritis, and bacteria. ${ }^{16,17}$ On the other hand, whether and how miRNA participates in the regulation of OP through osteoclast differentiation have been reported. ${ }^{18,19}$ Recent study has found that the expression of microRNA-151a-3p is reduced in prostate cancer cells. ${ }^{20}$ At present, there are few reports on the functions of microRNA$151 \mathrm{a}-3 \mathrm{p}$ in OP. The main goal of this study was to investigate the role of microRNA-151a-3p in OP, and our findings might provide insights into the novel drug targets for OP treatment.

\section{Methods}

\section{Clinical Samples}

This study was approved by the ethic board of Beijing Jishuitan Hospital. All participants signed the written informed consent. The experiments were carried out according to the declaration of Helsinki of 1964. A total of 15 postmenopausal osteoporosis patients (body mass index $22.7 \pm 6.2$, age range $57-80$ years old) were included in osteoporosis (OP) group. The patients were recruited with low-trauma fractures requiring surgical treatment (femoral neck, trochanter, and intertrochanteric region). Exclusion criteria included Patients with cancer, diabetic disease, heart disease, inflammatory, or metabolite disorder. Another 15 postmenopausal healthy females (BMI 25.8 \pm 3.7 , age range 61-76 years old) were selected as the control group.

\section{Cell Culture}

The murine macrophage cell-line RAW264.7 and the human monocyte cell-line THP-1 were provided by COBIOER (Nanjing, China). THP-1 and RAW264.7 cells were cultured in RPMI 1640 medium containing 10\% FBS (Jitai, Shanghai, China). RAW264.7 macrophages were treated by $50 \mathrm{ng} / \mathrm{mL}$ M-CSF (Andi, Shanghai, China) and $50 \mathrm{ng} / \mathrm{mL}$ nuclear factor-kappa receptor activator (RANKL; Lizhu, Beijing, China) to the differentiation of osteoclast. Finally, the cells were stained for the expression of TRAP using a commercially available kit (SigmaAldrich, St. Louis, MO).

\section{Cell Transfections}

The microRNA-151a-3p mimic, inhibitor, and the corresponding NCs were purchased from Ribobio (Guangzhou, China). For cell transfection, a total of $1 \times 10^{5}$ cells were transfected with $50 \mathrm{nM}$ microRNA-151a-3p mimic, inhibitor, or NCs by Lipofectamine 2000 reagent (Invitrogen).

\section{Bone Density Determination}

Bone mineral densities of lumbar spine were measured by dual-energy X-ray absorptiometry (DXA) with Hologic 4500 bone densitometer.

\section{Quantitative Real-Time PCR (RT-qPCR)}

Total RNAs were extracted from cells by TRIzol (Invitrogen, CA), and then was reverse-transcribed into cDNA using a RT kit (Thermo Fisher Scientific, USA). In the ABI-7300 system (Applied Biosystems; Thermo Fisher Scientific, Inc.), RT-qPCR was carried out by $\mathrm{ViiA}^{\mathrm{TM}} 7$ system (Jinuo, Shanghai, China). The thermocycling conditions were as follows: Pre-denaturation at $95^{\circ} \mathrm{C}$ for $2 \mathrm{~min}$, followed by 40 cycles of denaturation at $95^{\circ} \mathrm{C}$ for $15 \mathrm{sec}$ and annealing at $60^{\circ} \mathrm{C}$ for $45 \mathrm{sec}$, with final extension at $72^{\circ} \mathrm{C}$ for $10 \mathrm{~min}$. GAPDH and U6 were used as internal references. The expression of miRNA-151a-3p was detected by SYBR Premix Ex Taq II (Takara Biotechnology). ${ }^{21}$ The primers used are listed in Table 1. 
Table I Sequences of Primers Used in RT-qPCR

\begin{tabular}{|l|l|l|}
\hline Gene & Forward Primer (5'-3') & Reversed Primer (5'-3') \\
\hline $\begin{array}{l}\text { MicroRNA-15la-3p } \\
\text { U6 }\end{array}$ & $\begin{array}{l}\text { GGATGCTAGACTGAAGCTCCT } \\
\text { CTCGCTTCGGCAGCACA }\end{array}$ & $\begin{array}{l}\text { CAGTGCGTGTCGTGGAGT } \\
\text { AACGCTTCACGAATTTGCGT }\end{array}$ \\
\hline
\end{tabular}

\section{Animal Experiments}

The animal experiments were conducted under the Guide for the Care and Use of Laboratory Animals and were carried out following the institutional ethical principles for animal experiments. The experiments were approved by the Ethics Committee of Animal Usage Board of Beijing Jishuitan Hospital. Female Wistar rats (48 rats in total, $n=6$ per group) were obtained from the Animal Laboratory of the Jiangsu ALF Biotechnology Co., LTD (Jiangsu, China). Adaptive feeding was carried out in the laboratory animal center of the hospital for 2 weeks, and water and standard feed were taken freely. After intraperitoneal anesthesia, the abdomen of rats was fixed. The middle and lower parts of the back were routinely prepared, and disinfected with $3 \%$ iodophor. Under sterile conditions, the incision was made at a junction of about $1 \mathrm{~cm}$ under the costal margin and about $1 \mathrm{~cm}$ on both sides of the spine to separate the skin. The ovary was fully exposed, and the fat around the ovary was bluntly separated. After ligating the fallopian tubes and blood vessels, the ovaries were completely removed. The uterus was also placed in the abdominal cavity, layered sutured, and the incision was closed. The other side of the ovariectomy was performed in the same way. These rats received either antagomicroRNA-151a-3p or antago-NC through tail vein injection. The samples were collected after $30 \mathrm{~d}$ of tail injection. For euthanasia, animals were deeply anesthetized with sodium pentobarbital through intraperitoneal injection and sacrificed by cervical dislocation. Serum was collected for ELISA assays. The expression levels of RANKL, M-CSF, interleukin (IL)-1 $\alpha$, tumor necrosis factor (TNF)- $\alpha$, bone Gla protein (BGP) and type I collagen C-terminal peptide (CTX-I) in the serum were determined by enzyme-linked immunosorbent assay kit (Qincheng, Shanghai, China).

\section{Bone Densitometry}

The right femur with femoral head was fixed with $10 \%$ neutral formalin for $24 \mathrm{~h}$. After fully rinsing, the femur was decalcified by $10 \%$ EDTA and PBs for 3 to 4 weeks, and the fluid was changed every other day. After that, the longitudinal continuous section was made as the following steps: dehydration with ethanol gradient for $4 \mathrm{~h}$, transparency with xylene for $12 \mathrm{~h}$, immersion with soaking solution for $2 \mathrm{~h}$, embedding with paraffin for $4 \mathrm{~h}$. One section of each specimen was taken for HE staining for histological examination. Bone histomorphology analysis was performed with BI-2000 medical image analysis system. The average width of trabecular bone was calculated. Periosteal area percentage $(\%)=$ trabecular bone area/total tissue area $\times 100 \%$.

\section{Western Blotting}

Total protein was extracted from cells by RIPA lysis solution, and then was quantified using a BCA Kit. The same amount of total protein was separated by $10 \%$ SDS-PAGE and then transferred onto PVDF membranes. After being blocked with $5 \%$ skim milk for $1 \mathrm{~h}$ at room temperature, these membranes were incubated with anti-NFATc1 (1:500, Xibosheng, Shanghai, China), anti-c-Fos antibody (1:500, Xibosheng, Shanghai, China), anti-TRAP antibody (1:500, Xibosheng, Shanghai, China) and anti-GAPDH (1:1000, Xibosheng, Shanghai, China) overnight. After that, 1:5,000 labeled anti-rabbit secondary antibody was added. ${ }^{22}$ Finally, the protein was visualized using ECL reagent (Millipore, USA).

\section{Data Analysis}

The data were displayed as mean \pm standard deviation (SD). The analyses were performed in SPSS ver. 22.0. The normality of the distribution of continuous variables was assessed by Shapiro Wilk test. Multi-group data were compared by one-way ANOVA, followed by LSD test. The correlation between microR-151a-3p and BMD value was assessed by Pearson's correlation coefficient. $P<0.05$ was statistically significant.

\section{Results}

MicroRNA-I5Ia-3p Was Upregulated in Postmenopausal Osteoporotic Females and Negatively Related with Bone Mineral

\section{Density}

The characteristics of 30 subjects involved in this study are shown in Supplementary Table 1. There was no statistical difference in BMI value and age between OP group and the control group. The serum level of miRNA-151a-3p and the 

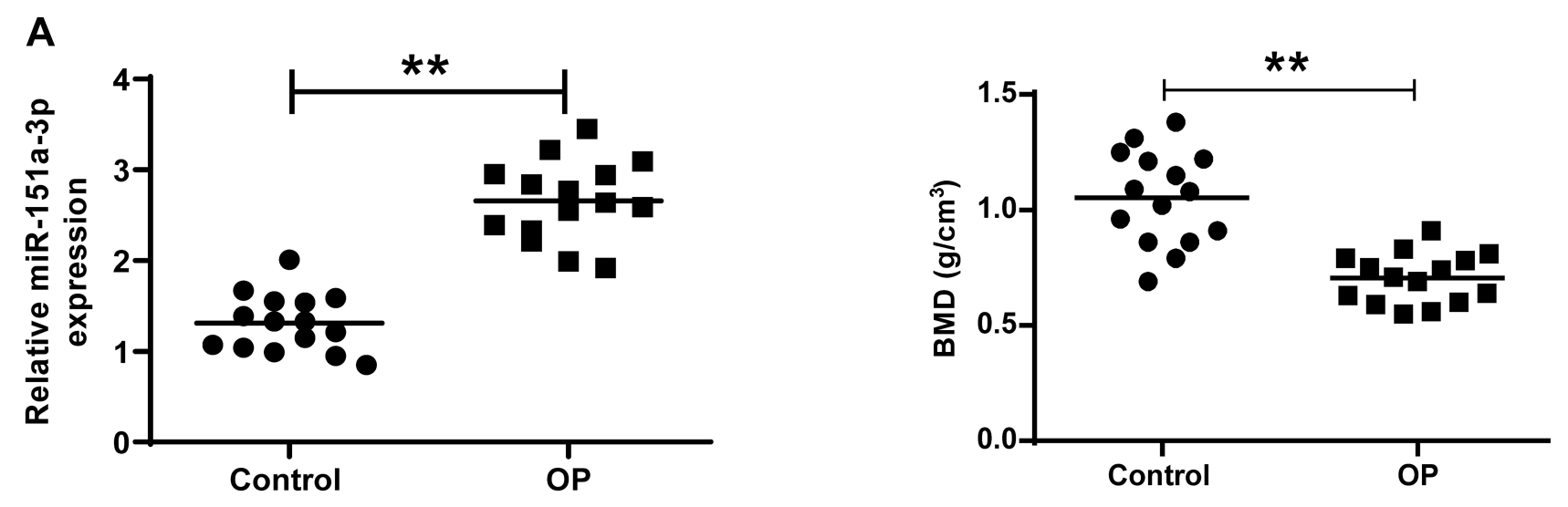

B

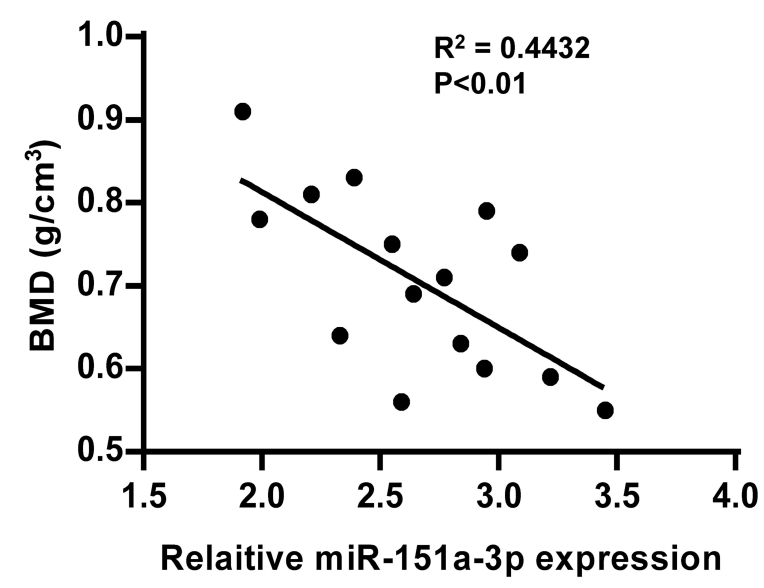

Figure I Patients with osteoporosis had increased microRNA-I5Ia-3p level and reduced BMD. (A) MicroRNA-I5Ia-3p level and BMD in patients with osteoporosis and control group were detected. (B) The correlation between microRNA-I5Ia-3p and BMD was analyzed by Pearson's correlation coefficient. $* * P<0.01$.

Abbreviation: BMD, bone mineral density.

bone mineral density (BMD) in both groups were detected. As shown in Figure 1A, microRNA-151a-3p level was remarkably higher in the serum of OP group than that in the serum of the control group $(P<0.01)$. Conversely, the BMD value in the OP group was significantly lower than that in the control group $(P<0.01)$. The correlation between microRNA-151a-3p and BMD value in OP group was assessed by Pearson's correlation coefficient. Results showed that microRNA-151a-3p level was negatively correlated with the BMD value (Figure 1B, $P<$ $0.01, \mathrm{R}^{2}=0.4432$ ).

\section{Overexpression of microRNA-15Ia-3p Improved RANKL-Induced Differentiation of RAW264.7 and THP-I Cells}

THP-1 and RAW264.7 cells were treated with RANKL and $\mathrm{M}-\mathrm{CSF}$ to induce osteoclast differentiation. As shown in
Figure 2A, TRAP-positive multinucleated cells with more than three nuclei were observed in Day 7 after stimulation. As shown in Figure 2B, the expression level of microRNA151a-3p was gradually increased in RANKL/M-CSF-induced THP-1 and RAW264.7 cells $(P<0.01)$, indicating that microRNA-151a-3p level was up-regulated during osteoclastogenesis. As shown in Figure 2C, in RANKL/M-CSFinduced HP-1 and RAW264.7 cells, the expression of microRNA-151a-3p was significantly up-regulated in the microRNA-151a-3p mimic group compared to that in the control group $(P<0.05)$. Furthermore, compared with the NC group, the expression levels of TRAP, NFATc1 and c-Fos were greatly raised in the microRNA-151a-3p mimic group $(P<0.01)$ (Figure 2D; Supplementary Figure 1A). These results demonstrated that overexpression of microRNA-151a$3 p$ improved RANKL-induced differentiation of THP-1 and RAW264.7 cells into osteoclasts. 

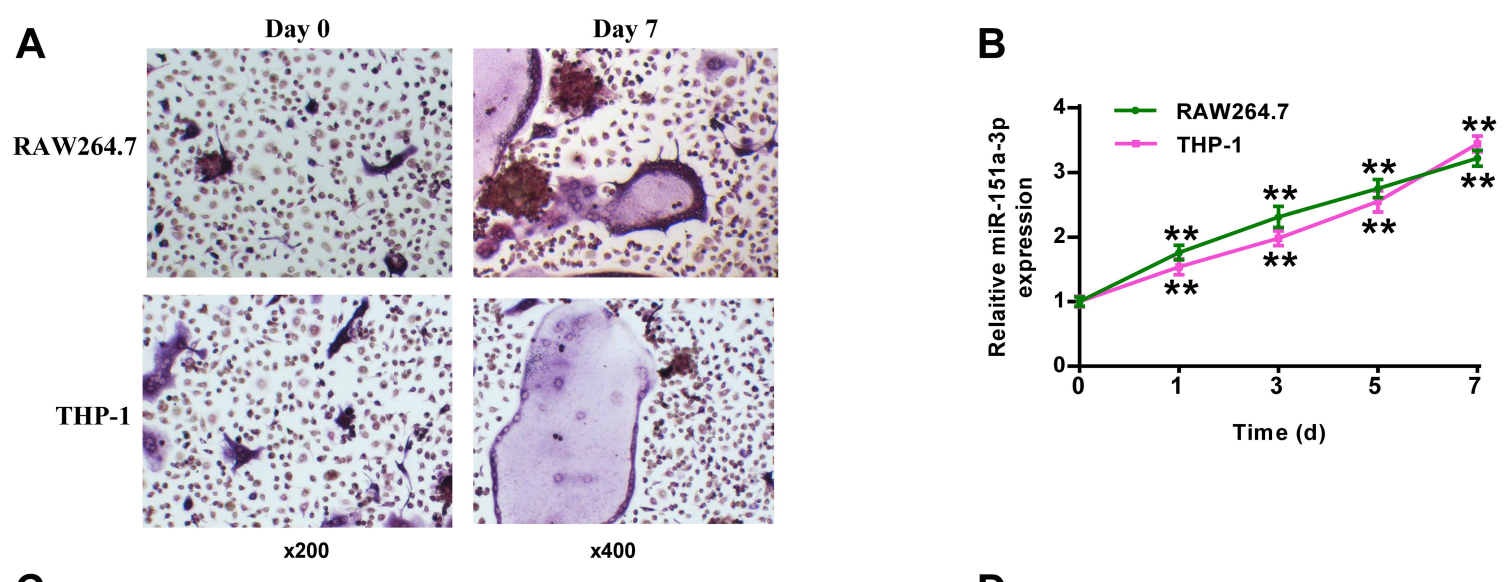

C
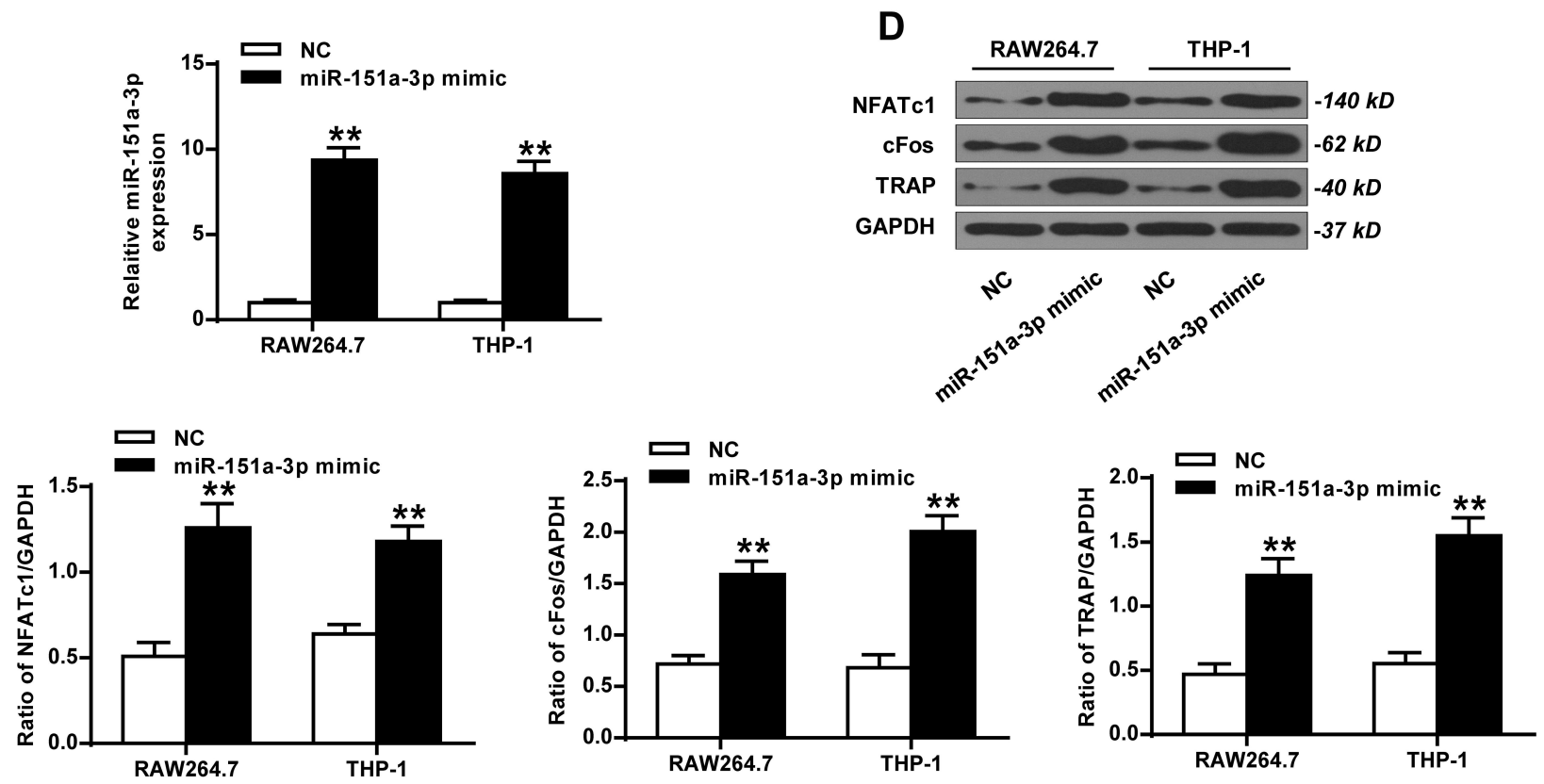

Figure 2 Overexpression of microRNA-15Ia-3p promoted RANKL-induced differentiation of THP-I and RAW264.7 cells into osteoclasts. (A and B) RAW264.7 and THPI cells were treated with RANKL and M-CSF. (A) The representative images of TRAP-stained cells. Magnification, x200 (Day 0) and x400 (Day 7). (B) The expression levels of microRNA-15Ia-3p in RANKL/M-CSF-induced cells. (C and D) RANKL/M-CSF-induced RAW264.7 and THP-I cells were treated with microRNA-I5Ia-3p mimic. (C) Expression levels microRNA-15Ia-3p in RAW264.7 and THP-I cells. (D) The expression levels of TRAP, NFATcl and c-Fos. $* * P<0.01$.

\section{Silencing of microRNA-I5la-3p Restrained RANKL-Induced}

\section{Differentiation of RAW264.7 and THP-I} Cells into Osteoclasts

As shown in Figure 3A, compared with $\mathrm{NC}$ inhibitor group, the expression levels of microRNA-151a-3p were significantly reduced in the microRNA-151a-3p inhibitor group $(P<0.05)$. Furthermore, compared with NC inhibitor group, the expression levels of TRAP, NFATc1 and c-Fos in the microRNA-151a-3p inhibitor group were reduced $(P<0.01)$ (Figure 3B; Supplementary Figure 1B). These results demonstrated that silencing of microRNA-151a-3p restrained RANKL-induced differentiation of RAW264.7 and THP-1 cells into osteoclasts.

\section{Silencing of microRNA-I 5 Ia-3p Altered the Osteoclastogenesis-Related Factors in OVX Rats}

The effect of microRNA-151a-3p on OP progression was further determined in vivo. As shown in Figure 4A, compared with sham group, the expression levels of microRNA-151a-3p were significantly raised in OVX rats, while the expression levels of microRNA-151a-3p were significantly decreased in OVX rats after transfection with antagomicroRNA-151a-3p, indicating successful transfection $(P<0.01)$. In addition, the expression levels of TNF- $\alpha$, RANKL, M-CSF, IL- $1 \alpha$ and CTX-I were significantly increased in OVX rats, and BGP levels were significantly decreased. After transfection with antagomicroRNA-151a-3p, the expression levels of TNF- $\alpha$, 

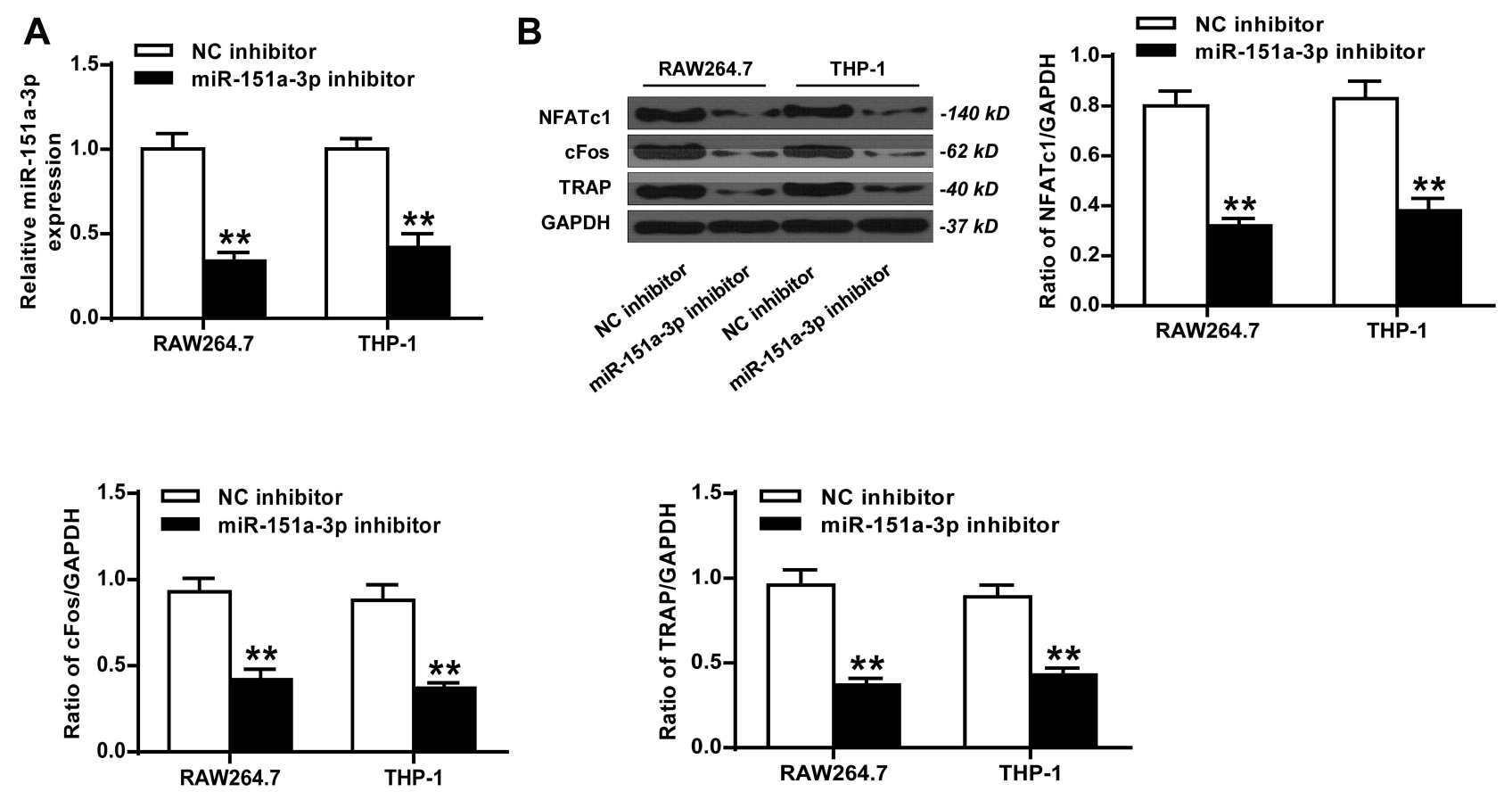

Figure 3 Silencing of microRNA-I5 Ia-3p restrained RANKL-induced differentiation of THP-I cells and RAW264.7 cells into osteoclasts. RAW264.7 and THP-I cells were treated with microRNA-I5Ia-3p inhibitor. (A) The expression levels of microRNA-I5 Ia-3p in THP-I cells and RAW264.7 cells. (B) The expression levels of TRAP, NFATcl and c-Fos. $* * p<0.01$.

RANKL, M-CSF, IL-1 $\alpha$ and CTX-I were significantly reduced in OVX rats, and BGP levels were significantly increased (Figure 4A-D). These results demonstrated that silencing of microRNA-151a-3p can regulate OP progression by affecting the osteoclastogenesis-related factors in OVX rat serum.

\section{Knockdown of microRNA-I 5 Ia-3p Increased Lumbar Spine BMD and Changed Bone Histomorphology in OVX Rat}

Compared with sham group, BMD values in OVX rats were significantly reduced. BMD values were significantly increased after transfection with antagomicroRNA-151a$3 p(P<0.05)$ (Figure 5A and D). As shown in Figure 5B and $\mathrm{C}$, compared with Sham group, BV/TV, Tb.Th and Tb. $\mathrm{N}$ values were significantly reduced in OVX rats. Tb.Th, $\mathrm{BV} / \mathrm{TV}$ and Tb.N values in OVX rats after transfection with antagomicroRNA-151a-3p were significantly increased $(P<0.01)$. These results demonstrated that knockdown of microRNA-151a-3p can attenuate bone loss in OVX rats.

\section{Discussion}

Osteoporosis is a systemic disorder of bone metabolism. ${ }^{23}$ Menopause is a natural physiological process, mainly due to ovarian function decline, cell apoptosis or programmed death, and decreased estrogen secretion. ${ }^{24}$ However, the pathogenesis of postmenopausal osteoporosis is not fully understood. Osteoporosis is mainly caused by the weakening of osteogenic differentiation and the enhancement of osteoclast differentiation. ${ }^{25}$ Osteoclasts are the dominant players in osteoclastosis. ${ }^{26}$ Osteoclasts are also the only cells in the body that can resorb bone matrix. It is also important to shape, repair and regulate calcium in the body. ${ }^{27,28}$ Therefore, osteoclasts are of great significance in patients with osteoporosis or other pathological osteoporosis.

Studies have found that microRNAs act as upstream regulators of multi-gene multi-target regulation. ${ }^{29,30}$ For example, microRNA-124 can regulate osteoclast differentiation by inhibiting the expression of NFATc1. ${ }^{31}$ MicroRNA-155 can simultaneously act on cytokine signaling inhibitory protein (SOCS1) and MITF to inhibit osteoclast differentiation. ${ }^{32}$ MicroRNA155 and microRNA-144-3p inhibit osteoclast differentiation. ${ }^{32,33}$ These studies indicate that miRNAs play important roles in osteoclast differentiation. MicroRNA-151a$3 p$ play a part in many diseases. ${ }^{34,35}$ Our study found that the expression of microRNA-151a-3p in the serum of osteoporosis women was significantly up-regulated, and the expression levels of microRNA-151a-3p were also gradually increased. In addition, the expression of microRNA-151a-3p in 

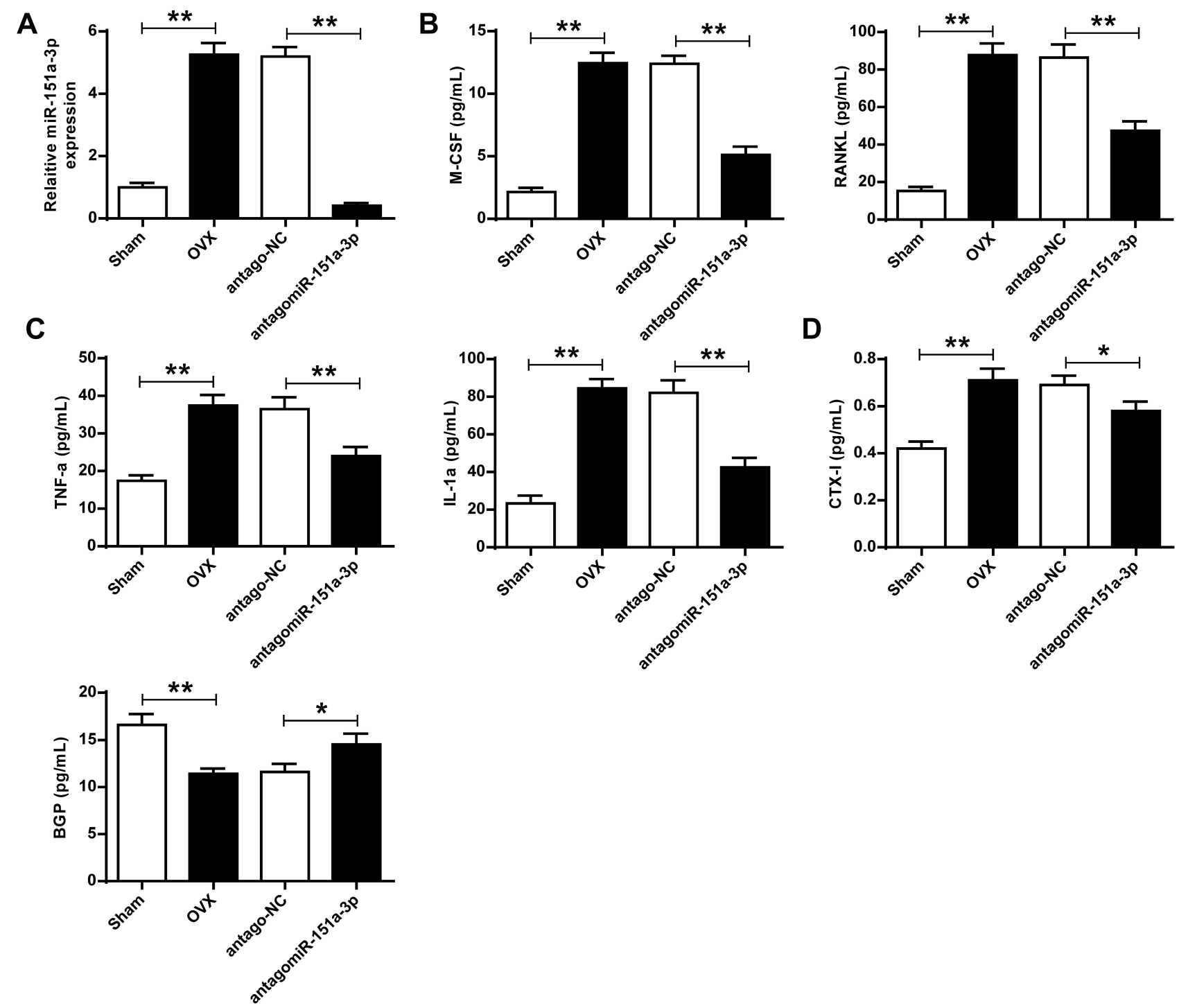

Figure 4 Silencing of microRNA-I5Ia-3p altered the expression levels of osteoclastogenesis-related factors in sera of OVX rats. OVX rats were treated with antago microRNA-I5Ia-3p or antago NC. (A) Expression levels of microRNA-I5Ia-3p was detected in different groups. The expression levels of M-CSF (B), RANKL (B), TNF- $\alpha$ (C), IL-I $\alpha$ (C), CTX-I (D), and BGP (D) in different groups. $N=6$ per group, $* P<0.05, * * P<0.0 I$.

postmenopausal women with osteoporosis was negatively correlated with BMD. Therefore, microRNA-151a-3p can be used as a target to control the development of OP.

NFATc1, c-Fos and TRAP are important transcription factors in the stage of osteoclast differentiation. ${ }^{36}$ The abnormal gene expression may cause serious osteoclastogenesis disorders. ${ }^{37}$ In this study, the expression levels of TRAP, c-Fos and NFATc1 were raised in the microRNA$151 \mathrm{a}-3 \mathrm{p}$ mimic group, indicating that microRNA-151a-3p can promote RANKL-induced the differentiation of osteoclasts.

There are two pathways for osteoclast proliferation, differentiation, and maturation, ${ }^{38}$ and the main pathway is the RANKL/RANK/OPG system, which has been expressed in the early stage of OP. ${ }^{39}$ The secondary pathway is the pathway of inflammatory factors. ${ }^{40}$ RANKL is the only factor currently found to induce osteoclast differentiation, development, and function. ${ }^{41}$ A large number of pro-inflammatory cytokines have been found to affect OP and promote the differentiation or activation of OP. ${ }^{42}$ Proinflammatory cytokines act directly or indirectly on OP through the RANK/RANKL/OPG system, TNF- $\alpha$ and IL-1 play key roles in the development of OP. ${ }^{43,44}$ In this study, after transfected with antagomicroRNA-151a-3p, the expression levels of TNF- $\alpha$, M-CSF, RANKL, IL- $1 \alpha$ and CTX-I were significantly decreased in OVX mice, and BGP levels were significantly increased. These results demonstrated that microRNA-151a-3p can regulate OP 
A

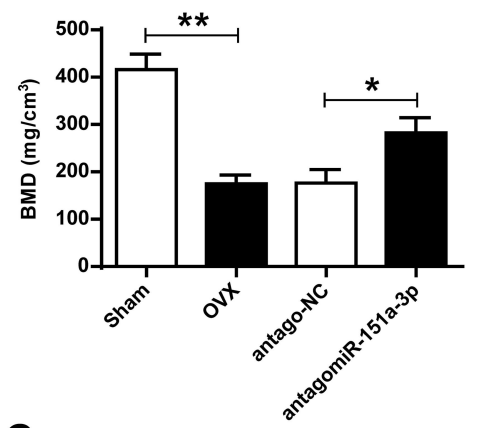

C

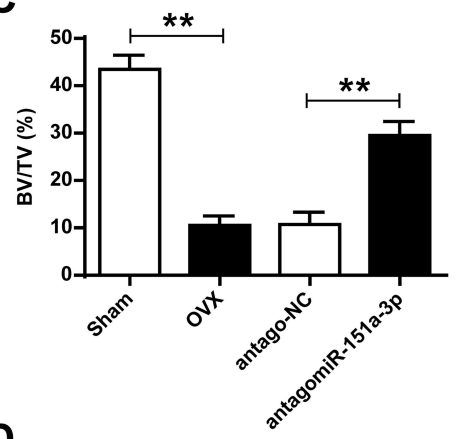

B

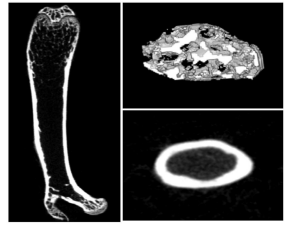

Sham

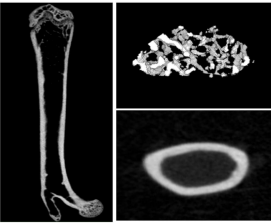

ovx

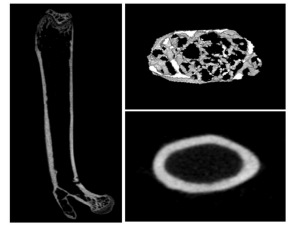

antago-NC

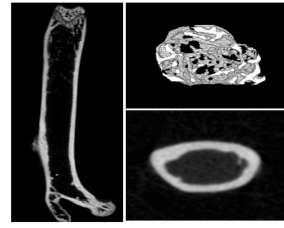

antagomiR-151a-3p

\section{D}

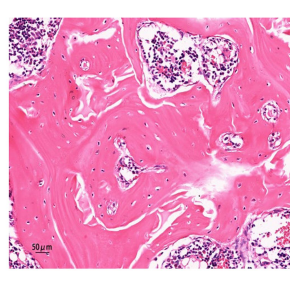

Sham

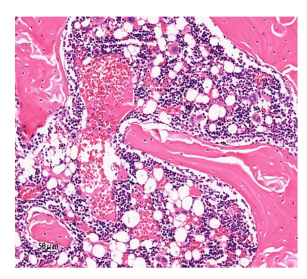

ovx
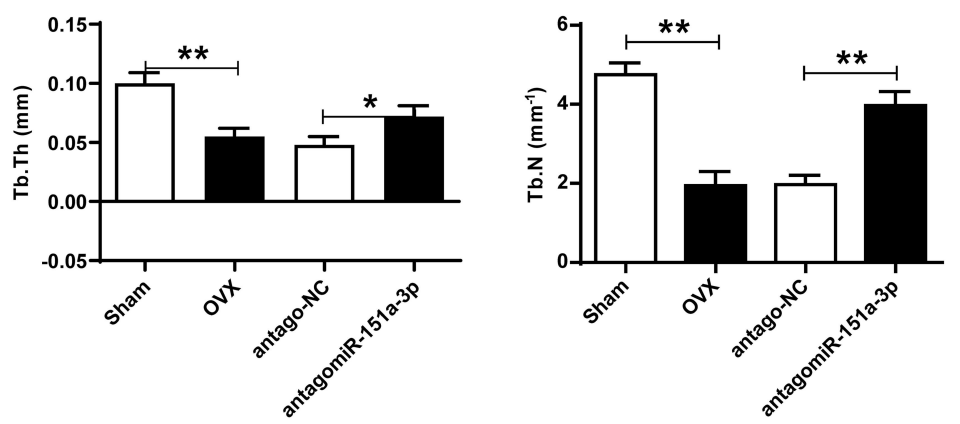

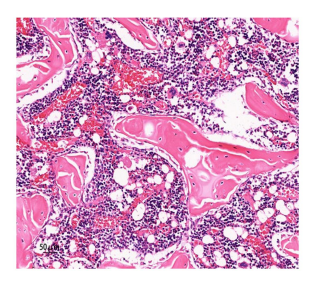

antago-NC

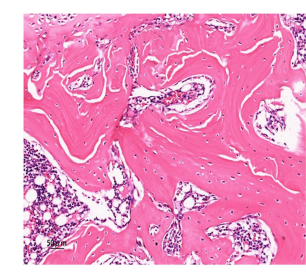

antagomiR-151a-3p

Figure 5 Knockdown of microRNA-I5Ia-3p increased BMD and altered bone histomorphology in OVX rats. OVX rats were treated with antago microRNA-I5Ia-3p or antago NC. (A) BMD was measured in different groups. (B and C) Bone histomorphometric parameters (BV/TV, Tb.Th, and Tb. N) were assessed. (D) Representative HE staining used to evaluate the pathological changes of osteogenic tissues in rats. $400 \times$, Bar $=50 \mu \mathrm{m}$. $\mathrm{N}=6$ per group, $* P<0.05, * * P<0.0 \mathrm{I}$.

Abbreviations: OVX, ovariectomized; BV/TV, bone volume fraction; Tb. Th, trabecular thickness; Tb. N, trabecular number.

by affecting osteoclastogenesis-related factors in OVX rat serum. It's well known that microRNAs can inhibit the expression of target genes by binding to complementary segments of messenger RNA and interfering with the formation of proteins by translation. ${ }^{39}$ Previous studies have found that miR-93-5p and miR-378 were involved in the regulation of OP by binding to BMP-2, a number of active bone morphogenetic proteins. ${ }^{46,47}$ Therefore, BMP-2 may play a critical role in miRNA-mediated regulation of OP. In our future study, we will further investigate whether microRNA-151a-3p functions in OP by directly binding to BMP-2. However, there were several limitations in our study. For instance, there is a risk of miRNA degradation in blood since tail vein injection is used as a delivery method for microRNAs and antagomirs. In addition, the tail vein injection was an unspecific method to target bone, which may introduce some bias into the study.

\section{Conclusion}

MicroRNA-151A-3P could partly regulate osteoporosis by promoting osteoclast differentiation. It is suggested that microRNA-151a-3p may be a potential pathogenic gene of OP, which provides experimental evidence for the clinical prognosis or targets of treatments in this disease.

\section{Abbreviations}

OP, osteoporosis; OC, osteoclasts; mi RNAs, microRNAs. 


\section{Data Sharing Statement}

The analyzed data sets generated during the study are available from the corresponding author on reasonable request.

\section{Ethics Approval and Consent to Participate}

The study was approved by the Beijing Jishuitan Hospital ethics committee and all patients signed informed consent. All procedures are carried out in accordance with the ethical standards of the Agency and/or the National Research Council. All patients and healthy volunteers provided written informed consent prior to their inclusion within the study.

\section{Author Contributions}

YHH designed the experiments in this study, and wrote the first draft of the article. All authors contributed to data analysis, drafting or revising the article, have agreed on the journal to which the article will be submitted, gave final approval of the version to be published, and agree to be accountable for all aspects of the work.

\section{Funding}

There is no funding to report.

\section{Disclosure}

The authors declare that they have no competing interests.

\section{References}

1. Giusti A, Papapoulos SE. Bisphosphonates for postmenopausal osteoporosis. Prim Metab Bone Dis Disord Min Metabolism. 2018: $545-552$.

2. Franic D, Verdenik IJ. Risk factors for osteoporosis in postmenopausal women - from the point of view of primary care gynecologist. Slov J Public Health. 2018;57(1):33-38. doi:10.2478/sjph-2018-0005

3. Mignot MA, Taisne N, Legroux I, Cortet B, Paccou JJ. Bisphosphonate drug holidays in postmenopausal osteoporosis: effect on clinical fracture risk. Osteoporos Int. 2017;28(12):3431-3438.

4. Wu F, Zhou D, Shen G, Cui Y, Lv Q, Wei FJC. Association of VDR and OPG gene polymorphism with osteoporosis risk in Chinese postmenopausal women. Climacteric. 2019;22:208-212.

5. Castrogiovanni P, Trovato FM, Szychlinska MA, Nsir H, Imbesi R, Musumeci G. The importance of physical activity in osteoporosis. From the molecular pathways to the clinical evidence. Histol Histopathol. 2016;31(11):1183-1194. doi:10.14670/HH-11-793

6. Pichler K, Loreto C, Leonardi R, Reuber T, Weinberg AM, Musumeci G. RANKL is downregulated in bone cells by physical activity (treadmill and vibration stimulation training) in rat with glucocorticoid-induced osteoporosis. Histol Histopathol. 2013;28 (9):1185-1196. doi:10.14670/HH-28.1185
7. Bandeira L, Bilezikian JP. Novel therapies for postmenopausal osteoporosis. Endocrinol Metab Clin. 2017;46(1):207. doi:10.1016/ j.ecl.2016.11.001

8. Radominski SC, Bernardo W, Paula APD, et al. Brazilian guidelines for the diagnosis and treatment of postmenopausal osteoporosis. Rev Bras Reumatol. 2017;57:S452-S466.

9. Migliaccio S, Fornari R, Greco EA, Luigi LD, Lenzi A. New therapeutical horizons in the management of postmenopausal osteoporosis. Aging Clin Exp Res. 2013;25(1):S117-S119. doi:10.1007/s40520-013-0106-x

10. Ma Y, Wang L, Zheng S, et al. Osthole inhibits osteoclasts formation and bone resorption by regulating NF- $\mathrm{KB}$ signaling and NFATc1 activations stimulated by RANKL. J Cell Biochem. 2019;120 (9):16052-16061. doi:10.1002/jcb.28886

11. Taguchi Y, Jin G, Inoue JI. NF-кB Signaling in Osteoclastogenesis. 2015.

12. Kim RY, Seong Y, Cho TH, Lee B, Kim IS, Hwang SJ. Local administration of nuclear factor of activated T cells (NFAT) c1 inhibitor to suppress early resorption and inflammation induced by bone morphogenetic protein-2. J Biomed Mat Res Part A. 2018;106 (5):1299-1310. doi:10.1002/jbm.a.36332

13. Li J, Li Y, Peng X, Li B, Hui Q, Chen YJ. In vivo analysis of the effects of CoCrMo and Ti particles on inflammatory responses and osteolysis. RSC Adv. 2018;8(10):5151-5157. doi:10.1038/s41598018-23465-5

14. Park K, Gu D, Jin S, et al. Pueraria lobate inhibits RANKL-mediated osteoclastogenesis via downregulation of CREB/PGC1 $/ \mathrm{c}-\mathrm{Fos} /$ NFATc1 signaling. Am J Chin Med. 2017;45(8):1-20.

15. Link JC, Hasin-Brumshtein Y, Cantor RM, et al. Diet, gonadal sex, and sex chromosome complement influence white adipose tissue miRNA expression. BMC Genomics. 2017;18(1):89. doi:10.1186/ s12864-017-3484-1

16. Zhang Z, Han Y, Sun G, et al. MicroRNA-325-3p inhibits cell proliferation and induces apoptosis in hepatitis B virus-related hepatocellular carcinoma by down-regulation of aquaporin 5. Cell Mol Biol Lett. 2019;24(1):1-15.

17. Zhu H, Cao XX, Liu J, et al. MicroRNA-488 inhibits endometrial glandular epithelial cell proliferation, migration, and invasion in endometriosis mice via Wnt by inhibiting FZD7. J Cell Mol Med. 2019;23:2419-2430.

18. Cao Z, Moore BT, Wang Y, et al. MiR-422a as a potential cellular microRNA biomarker for postmenopausal osteoporosis. PLoS One. 2014;9(5):e97098. doi:10.1371/journal.pone.0097098

19. Tang P, Xiong Q, Ge W, Zhang L. The role of microRNAs in osteoclasts and osteoporosis. RNA Biol. 2014;11(11):1355-1363. doi:10.1080/15476286.2014.996462

20. Zhang Y, Hao T, Zhang H, Wei P, Li X. Over-expression of miR-151a-3p inhibits proliferation and migration of PC-3 prostate cancer cells. Xi bao yu fen zi mian yi xue za zhi. 2018;34(3):247-252.

21. Zhang Y, Peng X, Yi L, et al. Evaluation of suitable reference genes for qRT-PCR normalization in strawberry (Fragaria $\times$ ananassa) under different experimental conditions. BMC Mol Biol. 2018;19 (1):1-10.

22. Swets M, Wouters A, Krijgsman D, et al. HLA-G protein expression in colorectal cancer evaluated by immunohistochemistry and Western blot analysis: its expression characteristics remain enigmatic. Clin Immunol. 2018;194:80-86. doi:10.1016/j.clim.2018.07.005

23. Yeon LY, Byul KH, Won LJ, et al. The association between urine albumin to creatinine ratio and osteoporosis in postmenopausal women with type 2 diabetes. $J$ Bone Metab. 2016;23(1):1-7.

24. Monteleone P, Mascagni G, Giannini A, Genazzani AR, Simoncini T. Symptoms of menopause - global prevalence, physiology and implications. Nat Rev Endocrinol. 2018;14(4):199-215. doi:10.1038/nrendo.2017.180 
25. Rao SS, Hu Y, Xie PL, et al. Omentin-1 prevents inflammation-induced osteoporosis by downregulating the pro-inflammatory cytokines. Bone Res. 2018;6(2):9. doi:10.1038/ s41413-018-0012-0

26. Li M, Luo R, Yang W, Zhou Z, Li C. miR-363-3p is activated by MYB and regulates osteoporosis pathogenesis via PTEN/PI3K/AKT signaling pathway. In Vitro Cell Dev Biol Animal. 2019;55 (5):376-386. doi:10.1007/s11626-019-00344-5

27. Löfvall H, Newbould H, Karsdal MA, et al. Osteoclasts degrade bone and cartilage knee joint compartments through different resorption processes. Arthritis Res Ther. 2018;20(1):67. doi:10.1186/s13075-018-1 564-5

28. Matsubara T, Kokabu S, Nakatomi C, et al. The actin-binding protein PPP1r18 regulates maturation, actin organization, and bone resorption activity of osteoclasts. Mol Cell Biol. 2018;38(4):e00425.

29. Jin D, Wu X, Yu H, et al. Systematic analysis of lncRNAs, mRNAs, circRNAs and miRNAs in patients with postmenopausal osteoporosis. Am J Translat Res. 2018;10(5):1498-1510.

30. Wong KC, Papaioannou AJ. Postmenopausal osteoporosis treatment update. Curr Treat Options Oncol. 2018;4(2):142-157.

31. Zhao N, Han D, Liu Y, et al. DLX3 negatively regulates osteoclastic differentiation through microRNA-124. Exp Cell Res. 2016;341 (2):166-176. doi:10.1016/j.yexcr.2016.01.018

32. Zhang $\mathrm{J}$, Zhao $\mathrm{H}$, Chen $\mathrm{J}$, et al. Interferon- $\beta$-induced miR-155 inhibits osteoclast differentiation by targeting SOCS1 and MITF. FEBS Lett. 2012;586(19):3255-3262. doi:10.1016/j.febslet.2012.06.047

33. Wang C, He H, Jiang Y, Wang L, Xu YJ. Reduced miR-144-3p expression in serum and bone mediates osteoporosis pathogenesis by targeting RANK. Biochem Cell Biol. 2018;96:627-635.

34. Mengelfrom J, Feddersen S, Halekoh U, et al. Circulating microRNAs disclose biology of normal cognitive function in healthy elderly people - a discovery twin study. J Hum Genet. 2018;26:1378-1387.

35. Perezsanchez C, Fontugalde P, Ruizlimon P, et al. Circulating microRNAs as potential biomarkers of disease activity and structural damage in ankylosing spondylitis patients. Hum Mol Genet. 2018;27 (5):875-890.

36. Kang MR, Jo SA, Yoon YD, et al. Agelasine D suppresses RANKLinduced osteoclastogenesis via down-regulation of c-Fos, NFATc1 and NF-кB. Marine Drugs. 2014;12(11):5643-5656. doi:10.3390/ md12115643
37. Perpétuo IP, Caetanolopes J, Vieirasousa E, et al. Ankylosing spondylitis patients have impaired osteoclast gene expression in circulating osteoclast precursors. Front Med. 2017;4:5.

38. Choi J, Choi SY, Lee SY, et al. Caffeine enhances osteoclast differentiation and maturation through p38 MAP kinase/Mitf and DC-STAMP/CtsK and TRAP pathway. Cell Signal. 2013;25 (5):1222-1227. doi:10.1016/j.cellsig.2013.02.015

39. Han SY, Kim YK. Berberine Suppresses RANKL-Induced Osteoclast Differentiation by Inhibiting c-Fos and NFATc1 Expression. Am J Chin Med. 2019;47(2):439-455. doi:10.1142/ S0192415X19500228

40. Qi DY, Perkins SL, Kling SJ, Russell RG. Divergent regulation of 1,25-dihydroxyvitamin D3 on human bone marrow osteoclastogenesis and myelopoiesis. J Cell Biochem. 1999;72(3):387395.

41. Chen K, Yuan Y, Wang Z, et al. Helvolic acid attenuates osteoclast formation and function via suppressing RANKL-induced NFATc1 activation. J Cell Physiol. 2019;234(5):6477-6488.

42. Wan H, Qian TY, Hu XJ, Huang CY, Yao WF. Correlation of serum CCL3/MIP-1 $\alpha$ levels with disease severity in postmenopausal osteoporotic females. Balkan Med J. 2018;35(4):320-325. doi:10.4274/ balkanmedj.2017.1165

43. Mamolini E, Cervellati C, Greco P, et al. VDR, RANKL and OPG polymorphisms as possible predisposing cofactors of postmenopausal osteoporosis: explorative study in Italian population. Gynecol Endocrinol. 2017;33:937-941.

44. Zha L, He L, Liang Y, et al. TNF- $\alpha$ contributes to postmenopausal osteoporosis by synergistically promoting RANKL-induced osteoclast formation. Biomed Pharmacother. 2018;102:369. doi:10.1016/ j.biopha.2018.03.080

45. Hanna J, Hossain GS, Kocerha J. The potential for microRNA therapeutics and clinical research. Front Genet. 2019;10:478. doi:10.3389/fgene.2019.00478

46. Qiao L, Li CG, Liu D. CircRNA_0048211 protects postmenopausal osteoporosis through targeting miRNA-93-5p to regulate BMP2. Eur Rev Med Pharmacol Sci. 2020;24(7):3459-3466. doi:10.26355/ eurrev_202004_20804

47. Liu S, Wang C, Bai J, et al. Involvement of circRNA_0007059 in the regulation of postmenopausal osteoporosis by promoting the microRNA-378/BMP-2 axis. Cell Biol Int. 2021;45(2):447-455. doi:10.1002/cbin.11502
Clinical Interventions in Aging

\section{Publish your work in this journal}

Clinical Interventions in Aging is an international, peer-reviewed journal focusing on evidence-based reports on the value or lack thereof of treatments intended to prevent or delay the onset of maladaptive correlates of aging in human beings. This journal is indexed on PubMed Central, MedLine, CAS, Scopus and the Elsevier
Bibliographic databases. The manuscript management system is completely online and includes a very quick and fair peer-review system, which is all easy to use. Visit http://www.dovepress.com/ testimonials.php to read real quotes from published authors. 